Martijn van Faassen, Anna van der Veen, Sonja van Ockenburg, Helma de Jong,

Elisabeth G. E. de Vries and Ido P. Kema*

\title{
Mass spectrometric quantification of urinary 6-sulfatoxymelatonin: age-dependent excretion and biological variation
}

https://doi.org/10.1515/cclm-2020-0455

Received April 6, 2020; accepted June 23, 2020; published online July 16,2020

\section{Abstract}

Objectives: Regulators of circadian rhythm, including melatonin, influence fundamental biological processes. Measuring the melatonin metabolite 6-sulfatoxymelatonin in urine can estimate melatonin production. 6-sulfatoxymelatonin is mainly analyzed by immunoassays, but these methods are hampered by cross-reactivity and poor reproducibility when used to analyze small molecules. Therefore, we validated a high-throughput liquid chromatography with tandem mass spectrometry (LC-MS/MS) method to quantify 6-sulfatoxymelatonin in urine. We evaluated age-dependent 24-h excretion of 6-sulfatoxymelatonin into urine and the biological variation of urinary excretion in healthy individuals.

Methods: The online solid phase extraction method combined with LC-MS/MS was validated according to international guidelines, and used to measure the excretion of 6-sulfatoxymelatonin into urine of 240 healthy individuals. Biological variation of 6-sulfatoxymelatonin excretion was examined in 10 healthy individuals.

Results: Urinary 6-sulfatoxymelatonin results were well within the validation criteria (interassay coefficient of variation: $<5.4 \%$, quantification limit: $0.2 \mathrm{nmol} / \mathrm{L}$ ). There was an age-related decrease in 6-sulfatoxymelatonin excretion into $24-\mathrm{h}$ urine $[\mathrm{F}(5$,

*Corresponding author: Ido Kema, PhD, Department of Laboratory Medicine, EA61 University Medical Center Groningen, P.O. Box 30.001, 9700 RB Groningen, The Netherlands, Phone: +31 50361 0399, Fax: +31 50361 9191, E-mail: i.p.kema@umcg.nl

Martijn van Faassen, Anna van der Veen and Helma de Jong: Department of Laboratory Medicine, University of Groningen, University Medical Center Groningen, Groningen, The Netherlands Sonja van Ockenburg: Department of Endocrinology, University of Groningen, University Medical Center Groningen, Groningen, The Netherlands

Elisabeth G. E. de Vries: Department of Medical Oncology, University Medical Center Groningen, University of Groningen, Groningen, The Netherlands
234)=13.9; $\mathrm{p}<0.001]$. Within-subject variation of 6-sulfatoxymelatonin was $39.2 \%$ in day urine, $15.1 \%$ in night urine, and $12.2 \%$ in 24-h urine. Between-subject variation was $39.1 \%$ in day urine, $37.9 \%$ in night urine, and $36.8 \%$ in $24-h$ urine.

Conclusions: This MS-based method enables straightforward, reproducible, and sensitive quantification of 6-sulfatoxymelatonin in urine. Urinary 6-sulfatoxymelatonin levels decreased with age. Biological variation of 6-sulfatoxymelatonin excretion into urine was high between subjects and lower within subjects, indicating that repeated measurements of 6-sulfatoxymelatonin in 24-h urine are needed in future studies.

Keywords: biological variation; melatonin; tandem mass spectrometry; 6-sulfatoxymelatonin; urine.

\section{Introduction}

Disturbed circadian rhythm and sleep is increasingly associated with the development of neurodegenerative, cardiovascular, and metabolic disorders [1, 2]. Melatonin is an important circadian regulator that signals the length of day and night. It also has antioxidant properties and modulates antioxidant enzymes and the immune system [3]. There is increasing interest in the mediating role of melatonin, since melatonin levels have been associated with breast and prostate cancer [4-6], myocardial infarction, and type 2 diabetes $[7,8]$. Reliable measurement of melatonin levels is important to substantiate these studies and elucidate the role of melatonin.

In humans, melatonin is metabolized by the liver to 6-hydroxymelatonin, $60-80 \%$ of which conjugates to sulfate to form 6 -sulfatoxymelatonin, and $\sim 10-20 \%$ of which conjugates to glucuronide. A small portion is excreted as 6-hydroxymelatonin [9, 10]. Measuring 6-sulfatoxymelatonin excretion into urine collected over $24 \mathrm{~h}$ (24-h urine) gives a reliable estimate of total daily melatonin production [11-13]. This is useful for fieldbased studies, where frequent sampling in blood or saliva 
is not practical [14]. To confirm the aforementioned association of urinary 6-sulfatoxymelatonin, the biological variation must also be determined. However, no data on the biological variation of 6-sulfatoxymelatonin excretion into urine is available. This information is relevant for the design and interpretation of epidemiological studies. Biological variation is an important element of the random measurement error [15]. Random measurement error can influence the association between a variable and outcome of interest, and can cause regression dilution bias $[15,16]$. Analytical variation is important when calculating the biological variation. To minimize analytical variation, measurements should be highly reproducible and free of interferences. Reliable methods are needed to achieve this.

Small molecules are preferably quantified by liquid chromatography in combination with tandem mass spectrometry (LC-MS/MS) because quantification of small molecules by immunoassays can be seriously flawed [17-20]. The purpose of the current study was to validate a reproducible, highly automated isotope dilution LC-MS/MS method for quantifying 6-sulfatoxymelatonin. We used this method to investigate the excretion of 6-sulfatoxymelatonin into urine in a large healthy population. We also determined biological variation of urinary 6-sulfatoxymelatonin excretion.

\section{Materials and methods}

\section{Reagents}

We obtained ULC-MS-grade methanol, acetonitrile, isopropanol, ammonium acetate, and formic acid from Biosolve BV (Valkenswaard, The Netherlands). Phosphate buffered saline (PBS; $0.01 \mathrm{M} \mathrm{Na}_{2} \mathrm{HPO}_{4}$, $1.8 \mathrm{mM} \mathrm{KH}_{2} \mathrm{PO}_{4}, 0.138 \mathrm{M} \mathrm{NaCl}, 27 \mathrm{mM} \mathrm{KCl}, \mathrm{pH} 7.4$ ) was prepared by the Department of Pharmacy of the UMCG. 6-sulfatoxymelatonin sodium salt was purchased from Toronto Research Chemicals Inc. (Toronto, Canada), and the $\mathrm{D}_{4}$-labeled internal standard (IS) for 6-sulfatoxymelatonin was purchased from CacheSyn Inc. (Mississauga, Canada). Ultrapure water was produced using an in-house purification system (Merck Millipore, MA, USA).

\section{Standard solutions and samples}

Working standard solutions containing 6-sulfatoxymelatonin as well as IS solutions were serially diluted from stock solutions with PBS.

Before analysis, $100 \mu \mathrm{L}$ aliquots of urine were pipetted into a $2.0 \mathrm{~mL}$ 96-deepwell plate (Greiner Bio-One, Kremsmünster, Austria). Subsequently, $50 \mu \mathrm{L}$ IS and $850 \mu \mathrm{L}$ PBS were added to all wells, and $50 \mu \mathrm{L}$ was injected into the online SPE LC-MS/MS system.

\section{Analyses and quantification}

A Symbiosis ${ }^{\mathrm{TM}}$ Pharma system (Spark Holland, Emmen, The Netherlands) was used for online solid phase extraction (SPE) and liquid chromatography as previously described [21]. Chromatography was performed using an Agilent Pursuit XRS Diphenyl column $(2.0 \times 150 \mathrm{~mm}, 3.0 \mu \mathrm{m})$ at $25{ }^{\circ} \mathrm{C}$. The binary gradient system was composed of $10 \mathrm{mM}$ ammonium acetate, $0.1 \%$ formic acid (FA) in water (eluent A), and $0.1 \% \mathrm{FA}$ in acetonitrile (eluent B). A linear gradient elution was performed, starting at $85 \% \mathrm{~A}, 15 \% \mathrm{~B}$, and in $2.5 \mathrm{~min}$ to $60 \% \mathrm{~A}$ and $40 \% \mathrm{~B}$. Then in $0.5 \mathrm{~min}$ to $25 \% \mathrm{~A}, 75 \% \mathrm{~B}$. After that, the column was flushed for 1 min. Finally, the column was equilibrated for $1.5 \mathrm{~min}$ at the starting gradient $(85 \% \mathrm{~A}, 15 \% \mathrm{~B})$ resulting in a total chromatographic run-time of $5.5 \mathrm{~min}$, which includes the online SPE extraction.

Mass spectrometry was performed with an XEVO TQ system (Waters, Milford, MA, USA) equipped with an electrospray source in positive ion mode. 6-sulfatoxymelatonin was analyzed in selected reaction monitoring mode (SRM). An overview of the SRM transitions, collision energies, and retention times for 6-sulfatoxymelatonin and its stable isotope is given in Supplemental Data, Table 1.

\section{Method validation}

The method was validated based on bioanalytical method validation guidelines from the European Medicines Agency [22].

Imprecision was determined by analyzing three urine pools with low, medium, or high 6-sulfatoxymelatonin concentrations 20 times on one day, and in replicate on 12 different days. The lower limit of quantification (LLOQ) for 6-sulfatoxymelatonin was determined by diluting a low-concentration urine sample with 6-sulfatoxymelatonin-free urine, and analyzing the dilutions on six different days. The LLOQ was $<20 \%$ precision and a signal to noise ratio of $>10$.

Recovery was assessed by spiking 6-sulfatoxy-melatonin concentrations in three different urine pools containing endogenous 6-sulfatoxymelatonin at three different levels. These samples were analyzed on five different days. The recovery percentage was calculated as follows: ([final concentration - initial concentration]/added concentration) $\times 100 \%$. Recovery between 85 and $115 \%$ was considered acceptable.

Results of the LC-MS/MS method were compared with those of a commercial ELISA (catalogue number RE54031, IBL International, Hamburg, Germany) by analyzing 40 residual 24-h urine samples collected during routine patient care. The ELISA was performed according to the vendor's instructions: $10 \mu \mathrm{L}$ urine, standard, and control samples were diluted with $500 \mu \mathrm{L}$ assay buffer, and $50 \mu \mathrm{L}$ of these diluted samples were pipetted into the microtiter plate wells. Then, $50 \mu \mathrm{L}$ of freshly prepared enzyme conjugate was pipetted into each well, followed by $50 \mu \mathrm{L}$ of antibody solution. The plate was covered with adhesive foil and incubated for $2 \mathrm{~h}$ on a plate shaker. The plate was then washed four times and $100 \mu \mathrm{L}$ substrate was added. Plates were then incubated for $30 \mathrm{~min}$ on a shaker. The reaction was stopped by adding $100 \mu \mathrm{L}$ of stop solution. The plate was briefly mixed and the optical density was measured at $450 \mathrm{~nm}$. Results were evaluated using Passing-Bablok and Bland-Altman regression. 


\section{Excretion study}

We used archived 24-h urine samples taken from healthy participants $(n=240)$ in a previous study [23]. Participants were randomly selected from the Lifelines Cohort Study [24]. Participants aged 20-80 years were included, and stratified for 20 males and 20 females per agedecade group. Participants were normotensive, had a normal blood count, and were not medicated. The BMI of participants was between 21 and $30 \mathrm{~kg} / \mathrm{m}^{2}$. For 24-h urine, the first sample was collected at $10 \mathrm{pm}$ and then all voided urine was collected until $10 \mathrm{pm}$ the next day. The collected urine was mixed and aliquots were stored at $-80{ }^{\circ} \mathrm{C}$ (collection of urine between 2009 and 2012) until analysis for 6-sulfatoxymelatonin by LC-MS/MS (in 2014). Long-term stability of 6-sulfatoxymelatonin in urine was previously shown to be at least 15 years at $-20^{\circ} \mathrm{C}$ [25]. The 24 -h excretion of 6-sulfatoxymelatonin (in nmol) was calculated as follows: 6-sulfatoxymelatonin concentration in 24-h urine $(\mathrm{nmol} / \mathrm{L}) \times$ volume of day urine $(\mathrm{L})$.

\section{Biological variation study}

Biological variation comprises the random variation of an analyte in one individual, the within-subject variation $\left(\mathrm{CV}_{\mathrm{i}}\right)$, the overall variation in different individuals, the between-subject variation $\left(\mathrm{CV}_{\mathrm{g}}\right)$, and the analytical variation $\left(\mathrm{CV}_{\mathrm{a}}\right)$. Biological variation was determined using urine samples collected during a previous longitudinal prospective observational study [26]. Ten adults between 18 and 65 years of age, who were free of medication and somatic and mental disease collected urine for 63 consecutive days. Urine was collected in two separate containers: a "night container" and a "day container". Participants were instructed to use the "night container" from the moment they went to bed until the first morning void. The "day container" contained all urine produced after the first morning void until the last void before going to bed. On each Wednesday, the biological variation of 6-sulfatoxymelatonin excretion in day, night, and 24-h urine samples was estimated. Nine samples from each participant were analyzed in duplicate in the same analytical run. The 24-h excretion of 6-sulfatoxymelatonin (in nmol) into urine was computed by combining the results of day and night urine as follows: (6-sulfatoxymelatonin concentration of night urine $[\mathrm{nmol} / \mathrm{L}] \times$ volume of night urine $[\mathrm{L}])+(6$-sulfatoxymelatonin concentration of day urine $[\mathrm{nmol} / \mathrm{L}] \times$ volume of day urine $[\mathrm{L}])$. Excretion of 6-sulfatoxymelatonin (in nmol) into day urine and night urine was computed as follows: 6-sulfatoxymelatonin concentration of day or night urine $\times$ volume of day or night urine. $\mathrm{CV}_{\mathrm{a}}, \mathrm{CV}_{\mathrm{i}}, \mathrm{CV}_{\mathrm{g}}$, reference change value (RCV), index of individuality (II), and homeostatic setting points for day urine, night urine, and 24-h urine were calculated as previously described [27, 28]. Quality specifications for analytical imprecision, bias and total error, expressed in percentages were calculated from the biological variation data for the $24 \mathrm{~h}$ urinary excretion of 6-sulfatoxymelatonin as follows: analytical imprecision < $0.5 \mathrm{CV}_{\mathrm{i}}$; bias $<0.25\left(\mathrm{CV}_{\mathrm{i}}{ }^{2}+\mathrm{CV}_{\mathrm{g}}{ }^{2}\right) \times 0.5$; total error $<k \times 0.5 \times \mathrm{CV}_{\mathrm{i}}+0.25$ $\left(\mathrm{CV}_{\mathrm{i}}^{2}+\mathrm{CV}_{\mathrm{g}}^{2}\right) \times 0.5$, being $k=1.65$ at $\alpha=0.05$ [27].

\section{Statistical analysis}

Statistical analyses were performed using SPSS version 23.0 for Windows (IBM Corporation, Chicago, IL, USA) and RStudio (Boston, MA, USA). Normally distributed data were expressed as mean \pm standard deviation (SD), and non-normally distributed data as median (interquartile range). A p-value of $<0.05$ (two-sided) was considered statistically significant. Differences in 6-sulfatoxymelatonin excretion between men and women were assessed by an independent-samples t-test, and differences between age decades were assessed by ANOVA with post-hoc Bonferroni correction. Non-parametric data were logarithmically transformed before analysis.

\section{Results}

\section{Method validation}

The total analysis time for one sample was $6 \mathrm{~min}$, which includes online SPE sample clean-up. 6-Sulfatoxymelatonin was eluted in $3.54 \mathrm{~min}$. Representative chromatograms for a standard solution and urine sample are shown in Figure 1.

Intraassay imprecision was $<2.5 \%$ and interassay imprecision was $<5.4 \%$ at the evaluated concentrations (Supplemental Data, Table 2). The LLOQ was $0.2 \mathrm{nmol} / \mathrm{L}$. Recovery for 6-sulfatoxymelatonin, as determined by a spike in concentration at three different levels, ranged from 90 to $115 \%$ (Table 2). No significant ion suppression was present in the region where 6-sulfatoxymelatonin eluted.

We compared the LC-MS/MS method with a commercial ELISA kit (IBL) using the Passing-Bablok equation. For ELISA, this was $0.47 \times \mathrm{LC}-\mathrm{MS} / \mathrm{MS}-3.2 ; \rho=0.846$. When the same calibrators were used in both assays, the equation was ELISA $=0.74 \times$ LC-MS/MS - 4.4; $\rho=0.838$ (Supplemental Data, Figure 1).

\section{Excretion study}

There was no difference in 6-sulfatoxymelatonin excretion between males and females [t (238) $=0.90, \mathrm{p}=0.37$ ], so further data analyses were performed on the whole group $(n=240)$. There was an age effect on 24-h 6-sulfatoxymelatonin excretion; excretion decreased with age $[F(5,234)=13.9$; p<0.001; Supplemental Data, Table 1; Figure 2]. The strength of the relationship as indexed by $\omega$ was 0.54 . 6-sulfatoxymelatonin output differed between age decades 20-29 and 50-59, 60-69 and 70-79, 30-39 and 50-59, 6069 and 70-79, 40-49 and 60-69, and 40-49 and 70-79 (Figure 2). Individuals who were 70-79 years old excreted $60 \%$ less 6-sulfatoxymelatonin on average than those who were 20-29 years old did (Table 1).

\section{Biological variation study}

One out of the 10 participants in the biological variation study (ID 3) was identified as an outlier based on results 

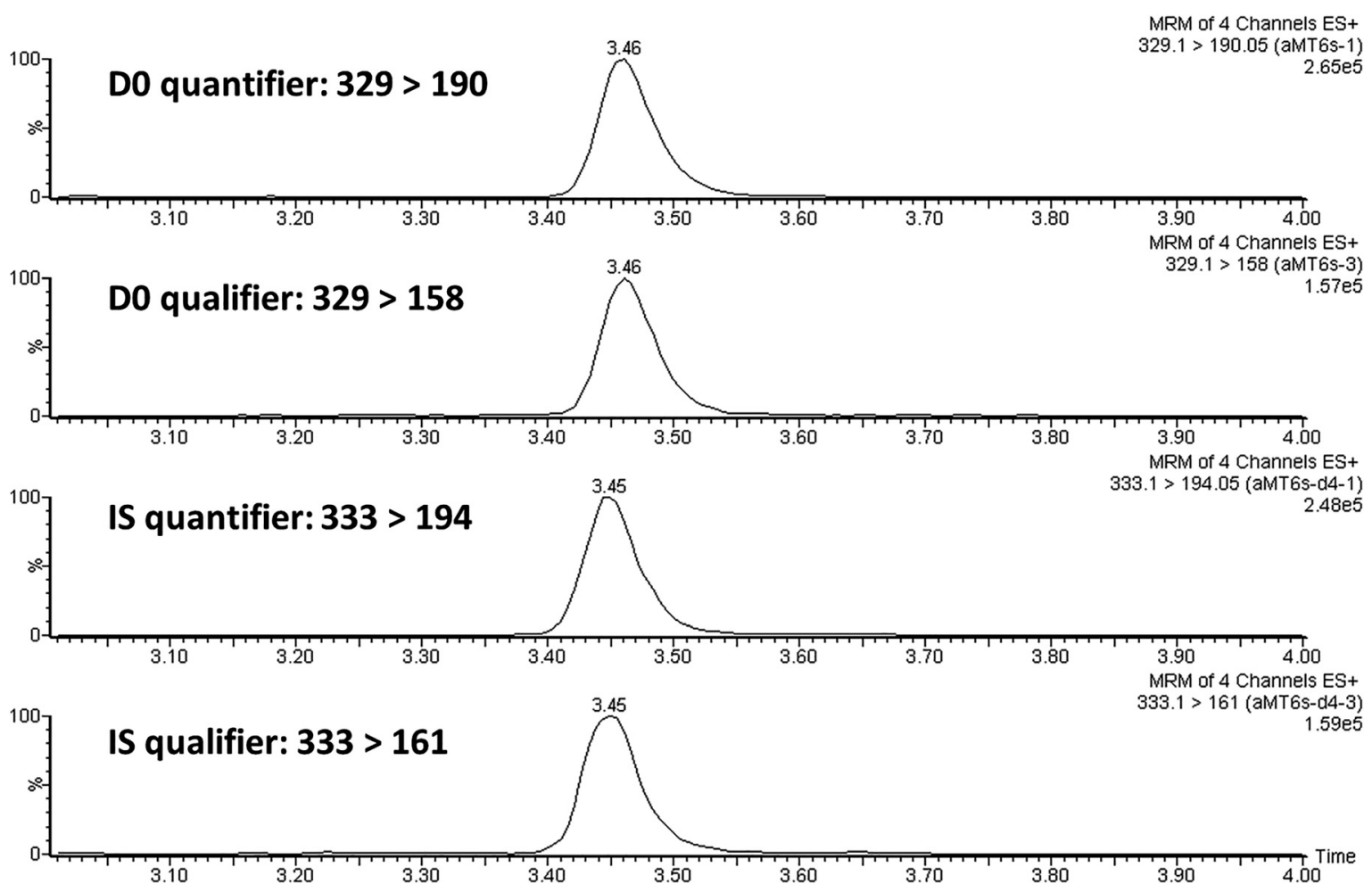

Figure 1: Representative chromatogram of 6-sulfatoxymelatonin measurements in urine.

Traces of 6- sulfatoxymelatonin are shown. $Y$-axis shows the relative intensity, where the $X$-axis shows the retention time in min. 6-sulfatoxymelatonin elutes at a retention time of 3.46 minutes. The concentration of 6- sulfatoxymelatonin in this urine sample was $27.5 \mathrm{nmol} / \mathrm{L}$.

from the Cochran and Dixon-Reed tests (Figure 3). Three males (average age, 27 years) and six females (average age, 34 years) were included (Supplemental Data, Table 3).

The average $\mathrm{CV}_{\mathrm{a}}$ of the LC-MS/MS method was 3.1\% (range, 1.6-4.7\%) for day urine, 1.7\% (range, 1.4-2.4\%) for night urine, and $1.6 \%$ (range, 1.1-2.2\%) for 24-h urine (Table 2). The $\mathrm{CV}_{\mathrm{a}}$ was higher in day urine as 6-sulfatoxymelatonin concentrations are lower during the day. The $\mathrm{CV}_{\mathrm{i}}$ for 6-sulfatoxymelatonin was highest in day urine (average 39.2\%; 95\% confidence interval [CI], 28-49). The $\mathrm{CV}_{\mathrm{i}}$ was $15.1 \%$ for night urine $(95 \% \mathrm{CI}, 11.8-18.3)$, and
$12.2 \%$ for 24 -h urine ( $95 \% \mathrm{CI}$, 8.6-15.9). The $\mathrm{CV}_{\mathrm{g}}$ was $39.1 \%$ in day urine, $37.9 \%$ in night urine, and $36.8 \%$ in $24-\mathrm{h}$ urine. The RCV (the relative change of serial measurements) was 109\% for day urine, $42 \%$ for night urine, and 34\% for 24-h urine. Furthermore, the II was $<1.0$ for day urine, night urine, and 24-h urine, suggesting that population-based reference intervals are not valid for measuring urinary 6-sulfatoxymelatonin. An average of nine samples of night urine, 59 samples of day urine, and six samples of 24-h urine were needed to determine the individual homeostatic set point with 10\% accuracy (Table 2). The analytical performance

Table 1: Urinary 6-sulfatoxymelatonin excretion over $24 \mathrm{~h}$ in healthy individuals divided by age decade, $\mathrm{n}=40$ individuals per decade.

\begin{tabular}{|c|c|c|c|c|c|c|}
\hline Decade & $20-29$ & $30-39$ & $40-49$ & $50-59$ & $60-69$ & $70-79$ \\
\hline 6-Sulfatoxymelatonin, $\mathrm{nmol} / 24 \mathrm{~h}^{\mathrm{a}}$ & $47.7[30-71]$ & $48.7[28-62]$ & $32.8[21-48]$ & $23[16-36]$ & $21[12-30]$ & $19[9.2-30]$ \\
\hline$\%$ Change to $20-29$ decade & & +2 & -31 & -52 & -56 & -60 \\
\hline \multicolumn{7}{|l|}{ One-way ANOVA ${ }^{\mathrm{b}}$} \\
\hline $20-29$ & - & - & - & - & - & \\
\hline $30-39$ & 1.0 & - & - & - & - & \\
\hline $40-49$ & 0.87 & 1.0 & - & - & - & \\
\hline $50-59$ & 0.003 & 0.009 & 1.0 & - & - & - \\
\hline $60-69$ & $<0.001$ & $<0.001$ & 0.013 & 1.0 & - & \\
\hline $70-79$ & $<0.001$ & $<0.001$ & $<0.001$ & 0.14 & 1.0 & \\
\hline
\end{tabular}

${ }^{\mathrm{a}}$ Values are represented as median [interquartile range]. ${ }^{\mathrm{b}}$ Post-hoc analysis with Bonferroni correction. 
Table 2: Biological variation of 6-sulfatoxymelatonin excretion into urine.

\begin{tabular}{|c|c|c|c|c|c|c|c|c|c|}
\hline & \multirow[t]{2}{*}{$\mathrm{CV}_{\mathrm{a}}$} & \multirow[t]{2}{*}{$\mathrm{CV}_{\mathrm{i}}^{\mathrm{a}}$} & \multirow[t]{2}{*}{$\mathrm{CV}_{\mathrm{g}}$} & \multirow[t]{2}{*}{ II } & \multirow[t]{2}{*}{ RCV (\%) } & \multicolumn{3}{|c|}{ RCV log normal } & \multirow[t]{2}{*}{ Samples required } \\
\hline & & & & & & + & - & Mean & \\
\hline Day urine, nmol & 3.1 & $39.2(28-49)$ & 39.1 & 0.91 & 109 & 201 & -63 & 132 & 59 \\
\hline Night urine, nmol & 1.7 & $15.1(12-18)$ & 37.9 & 0.40 & 42 & 53 & -34 & 43 & 9 \\
\hline 24-h urine, nmol & 1.6 & $12.2(8.6-16)$ & 36.8 & 0.33 & 34 & 42 & -28 & 35 & 6 \\
\hline
\end{tabular}

${ }^{\mathrm{a}} \mathrm{CV}_{\mathrm{i}}$ with $95 \%$ confidence interval; ${ }^{\mathrm{b}} \mathrm{To}$ estimate homeostatic setting point with $10 \%$ accuracy with $95 \%$ confidence. $\mathrm{CV}_{\mathrm{a}}$, analytical variation; $\mathrm{CV}_{\mathrm{i}}$, within-subject variation; $\mathrm{CV}_{\mathrm{g}}$, between-subject variation; II, index of individuality; $\mathrm{RCV}$, reference change value; $24 \mathrm{~h}, 24 \mathrm{~h}$.

requirements for imprecision, accuracy and total allowable error for the 24-h excretion of 6-sulfatoxymelatonin in urine were as follows: $6.1,9.7$, and $19.8 \%$, respectively.

\section{Discussion}

This study shows that online SPE in combination with LCMS/MS can rapidly detect 6-sulfatoxymelatonin in urine samples in a sensitive and reproducible manner. This is the first attempt to quantify the biological variation of urinary 6-sulfatoxymelatonin excretion, and high between-subject variation was revealed. Excretion of 6-sulfatoxymelatonin into 24-h urine had the lowest within-subject variation.

There is a clear need for this reproducible and potentially traceable analysis method, given the rising interest in melatonin [29]. The most frequently used method for measuring 6-sulfatoxymelatonin is the radioimmunoassay or enzyme-linked immunoassay [30]. Although immunoassays are easy to use and provide valuable information, LC-MS/MS is the preferred method for analyzing small molecules [18, 31]. This novel LC-MS/MS method performed very well in our validation study. Compared with the previous study that used LC-MS/MS to detect 6-sulfatoxymelatonin in urine, our method showed improved interassay precision and a lower LOQ [32]. Our method fulfilled the analytical performance requirements for imprecision derived from the biological variation, as our interassay imprecision was below the desired imprecision of $6.1 \%$ at all concentrations tested.

Our study showed an age-related decline of 6-sulfatoxymelatonin excretion into 24 -h urine. Individuals aged 40-49 years excreted 31\% less 6-sulfatoxymelatonin than individuals who were $20-29$ or 30-39 years old. This was a gradual age-related decline (Supplemental Data, Figure 2). The oldest participants (70-79 years old) excreted $60 \%$ less 6-sulfatoxymelatonin into their urine than those aged 20-29 years. Other studies have also shown a gradual age-related decline, although this was less than the difference reported in the present study [33, 34]. There are two potential explanations for this disparity between studies. The first is different definitions of the healthy population.

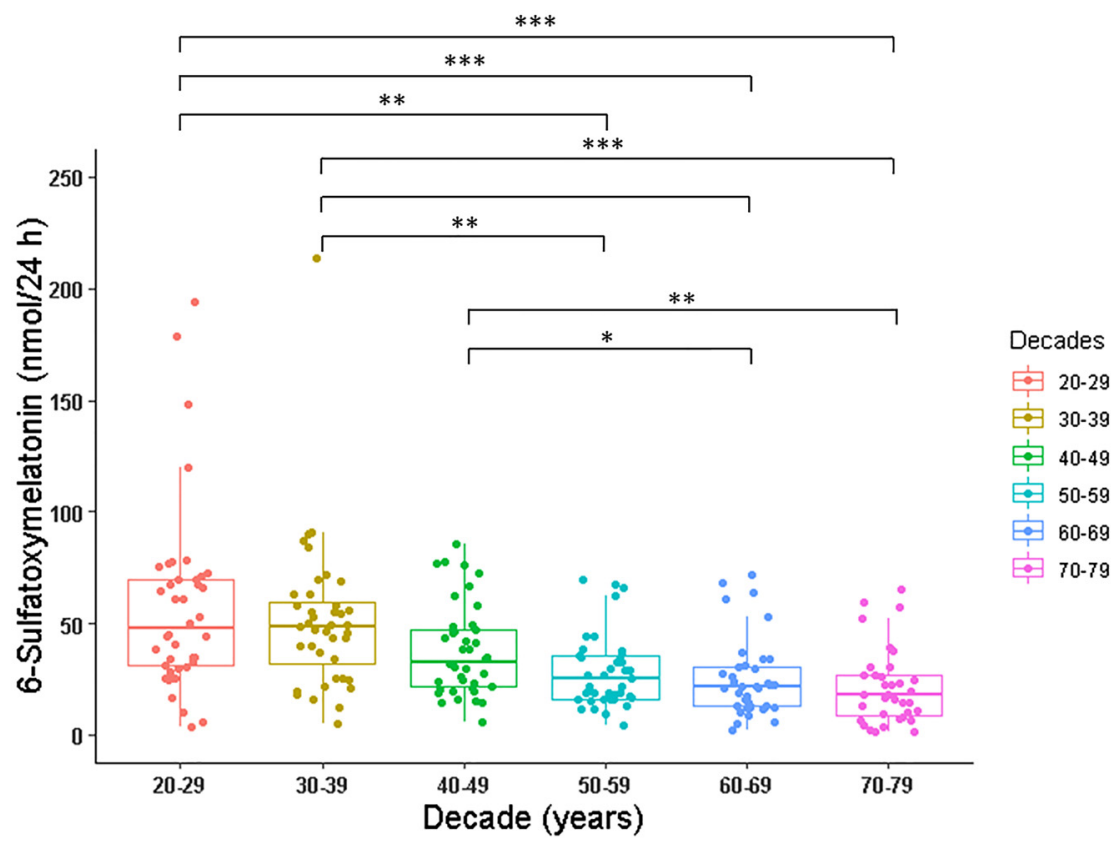

Figure 2: Box plot of 6-sulfatoxymelatonin excretion in 24-h urine samples from healthy individuals aged between 20 and 80 years, divided by decades $(n=40$ individuals per decade). One-way ANOVA with posthoc analysis with Bonferroni correction; ${ }^{*} p<0.05$; ${ }^{\star *} p<0.01$; $\star \star \star p<0.001$. Abbeviation: h, hour. 
A

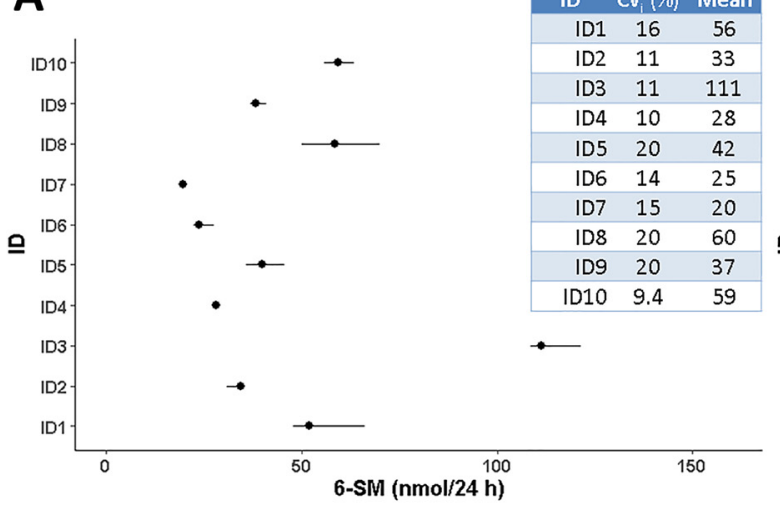

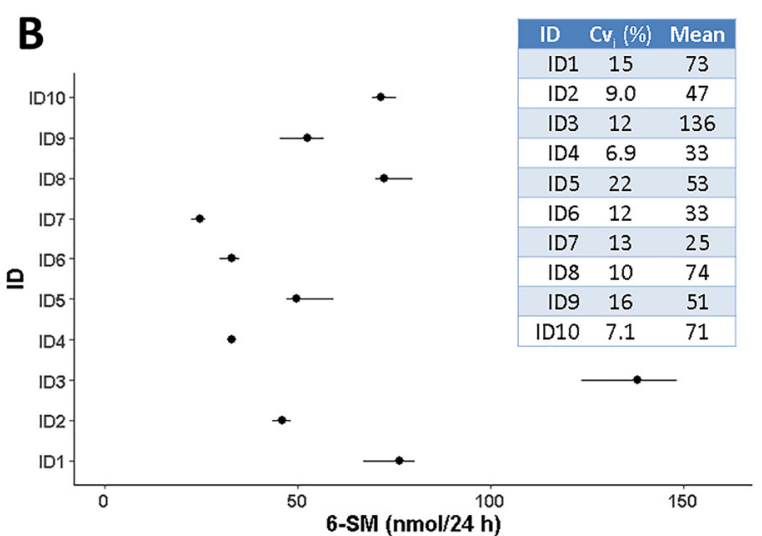

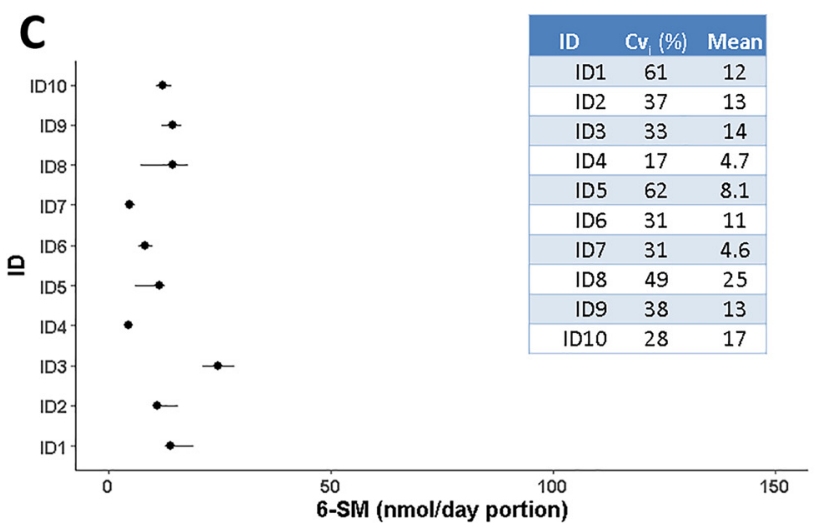

Figure 3: (A) 6-sulfatoxymelatonin excretion into night urine; (B) 6-sulfatoxymelatonin excretion into 24-h urine; (C) 6-sulfatoxymelatonin excretion into day urine portion for each individual.

On the $y$-axis, the individual participants (ID1-ID10) are shown. Median (solid dot) and interquartile range (horizontal line) are shown for each participant. Inserted tables show the CVi (\%) and mean value for 6-sulfatoxymelatonin for each participant. ID3 was identified as an outlier, determined by results from either Cochran (CVi) or DixonReed (CVg) tests and was omitted from further analyses. Abbreviations: 6-SM, 6sulfatoxymelatonin; 24 h, 24 hour; CVi, within-subject variation; CVg, between-subject variation.

The two previous studies did not have clear criteria for selecting healthy individuals. In contrast, we performed a physical examination and used a questionnaire when selecting our healthy participants [24]. We also had clearly defined inclusion criteria: only individuals who were normotensive, unmedicated, and had a BMI of $21-30 \mathrm{~kg} / \mathrm{m}^{2}$ were included. Second, previous studies used immunoassays, which have higher analytical variation and interferences, which can influence the outcome [33, 34]. We analyzed if the 6-sulfatoxymelatonin excretion correlated with the length of day (collection date of urine was used for this), to see if the excretion pattern was affected by seasonal changes. No correlation was found between length of day and 6-sulfatoxymelatonin excretion (data not shown). Our study substantiates previous reports of extreme variability between individuals of the same age (Figure 2) [33, 34]. However, as we only used single $24 \mathrm{~h}$ urine per individual no firm conclusions can be drawn from our study about the decline of urinary 6-sulfatoxymelatonin with age. Longitudinal studies with multiple samples per individual are needed to clarify whether the observed decline of urinary 6-sulfatoxymelatonin excretion with age is causal or not.

Biological variation is the natural fluctuation of bodyfluid constituents, and is used to diagnose and follow-up disease as well as to set analytical specifications in the clinical laboratory [35]. Our findings have revealed a large between-subject variation in urinary 6-sulfatoxymelatonin excretion. However, there was less biological variation within the same subject.

This is the first study to investigate the within-subject and between-subject biological variation of urinary 6-sulfatoxymelatonin excretion. This means that we cannot compare our findings with those of previous studies, as these studies did not correct for analytical variation, which is a prerequisite for estimating biological variation. Previous studies have reported a $13.7-17.4 \%$ intra-individual variation for 6-sulfatoxymelatonin excretion in 24-h urine, which is similar to the within-subject variation of $12.2 \%$ reported in the current study [12, 36-38]. Notably, 6-sulfatoxymelatonin 


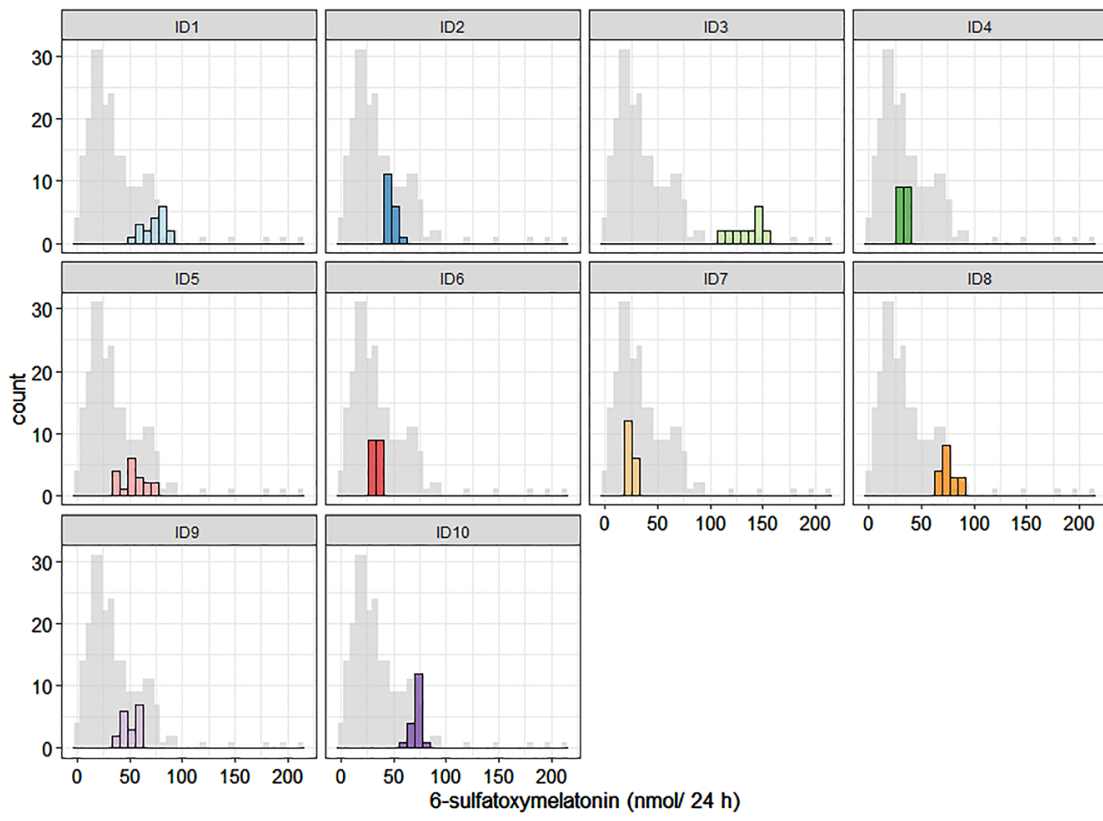

Figure 4: Histogram of the within-subject variation of 6-sulfatoxymelatonin excretion into 24-h urine in ten individuals from the biological variation study (ID1 - ID10) against 6-sulfatoxymelatonin excretion into 24-h urine of 240 healthy individuals from the excretion study plotted in the background (grey shaded area). Abbreviation: $\mathrm{h}$, hour. excretion into 24-h urine had a lower within-subject variation than 6-sulfatoxymelatonin excretion into night urine. Many studies have only used urine from the first morning void to represent 6-sulfatoxymelatonin excretion, as this is more convenient for the participant. Previous research has shown that this reflects approximately $80 \%$ of total 6-sulfatoxymelatonin excretion, which agrees with our finding that average 6-sulfatoxymelatonin excretion is similar in night urine and 24-h urine ( 47.2 vs. $59.5 \mathrm{nmol})$ [12, 39]. However, we revealed higher variability in results obtained using night urine samples. Using urine from the first morning void likely introduces even more variation, as possible urine voids during the night are missed. Collecting all urine over $24 \mathrm{~h}$ is a more reliable estimate of 6-sulfatoxymelatonin excretion. Figure 4 illustrates the high between-subject variation for 6-sulfatoxymelatonin excretion into 24-h urine. This finding emphasizes that epidemiological studies using 6-sulfatoxymelatonin excretion as a variable should use 24-h urine, take repeated measurements, and apply a precise analysis method. This will improve estimates of the true values. Increasing the sample size will only make estimates more precisely incorrect [15].

Caution is advised when drawing conclusions from single measurements of 6-sulfatoxymelatonin excretion. This could explain the contradicting results of two metaanalyses examining the association of 6-sulfatoxymelatonin excretion and breast cancer [40, 41]. Although withinsubject variation is much lower than between-subject variation in measurements of 6-sulfatoxymelatonin excretion into $24-\mathrm{h}$ urine, there is still considerable difference in within-subject variation between the participants. For example, some participants show only $7 \%$ variation, whereas others show around 20\% variation (Figures 3B and 4). To what extent this variation is caused by factors like variation in sleeping behavior or light exposure remains to be established. Within-subject variation may be lower under controlled laboratory conditions [42].

Future prospective cohort studies investigating the role of 6-sulfatoxymelatonin should use well-validated, precise methods to improve estimates. In addition, subjects should be their own control, and urine should be collected over $24 \mathrm{~h}$ at six different time points to accurately determine an individual's setting point.

Acknowledgments: We would like to acknowledge the excellent technical assistance of Maria Schenk.

Research funding: The biological variation study was financed with a bench fee granted by the Junior Scientific Masterclass program at the University Medical Center Groningen and University of Groningen.

Author contributions: All authors have accepted responsibility for the entire content of this manuscript and approved its submission.

Competing interests: Authors state no conflict of interest. Informed consent: Informed consent was obtained from all individuals included in this study.

Ethical approval: Research involving human subjects complied with all relevant national regulations, institutional policies and is in accordance with the tenets of the Helsinki Declaration (as revised in 2013), and has been approved by the authors' Institutional Review. 


\section{References}

1. Abbott SM, Knutson KL, Zee PC. Health implications of sleep and circadian rhythm research in 2017. Lancet Neurol 2018 Jan 1;17: 17-8.

2. Potter GDM, Skene DJ, Arendt J, Cade JE, Grant PJ, Hardie LJ. Circadian rhythm and sleep disruption: causes, metabolic consequences, and countermeasures. Endocr Rev 2016 Dec;37: 584-608.

3. Hardeland R. Melatonin and the pathologies of weakened or dysregulated circadian oscillators. J Pineal Res 2017 Jan;62:e12377.

4. Devore EE, Warner ET, Eliassen AH, Brown SB, Beck AH, Hankinson SE, et al. Urinary melatonin in relation to postmenopausal breast cancer risk according to melatonin 1 receptor status. Cancer Epidemiol Biomarkers Prev 2017 Mar;26: 413-9.

5. Nooshinfar E, Safaroghli-Azar A, Bashash D, Akbari ME. Melatonin, an inhibitory agent in breast cancer. Breast Cancer 2017 Jan 26;24:42-51.

6. Sigurdardottir LG, Markt SC, Rider JR, Haneuse S, Fall K, Schernhammer ES, et al. Urinary melatonin levels, sleep disruption, and risk of prostate cancer in elderly men. Eur Urol 2015 Feb;67:191-4.

7. McMullan CJ, Rimm EB, Schernhammer ES, Forman JP. A nested case-control study of the association between melatonin secretion and incident myocardial infarction. Heart 2017 May; 103:694-701.

8. Tuomi T, Nagorny CLF, Singh P, Bennet H, Yu Q, Alenkvist I, et al. Increased melatonin signaling is a risk factor for type 2 diabetes. Cell Metab 2016 Jun 14;23:1067-77.

9. Kopin IJ, Pare CM, Axelrod J, Weissbach H. The fate of melatonin in animals. J Biol Chem 1961 Nov;236:3072-5.

10. Jones RL, McGeer PL, Greiner AC. Metabolism of exogenous melatonin in schizophrenic and non-schizophrenic volunteers. Clin Chim Acta 1969 Nov;26:281-5.

11. Fellenberg AJ, Phillipou G, Seamark RF. Measurement of urinary production rates of melatonin as an index of human pineal function. Endocr Res 1980;7:167-75.

12. Bojkowski CJ, Arendt J, Shih MC, Markey SP. Melatonin secretion in humans assessed by measuring its metabolite, 6-sulfatoxymelatonin. Clin Chem 1987 Aug;33:1343-8.

13. Nowak R, McMillen IC, Redman J, Short RV. The correlation between serum and salivary melatonin concentrations and urinary 6-hydroxymelatonin sulphate excretion rates: two noninvasive techniques for monitoring human circadian rhythmicity. Clin Endocrinol (Oxf) 1987 Oct;27:445-52.

14. Benloucif S, Burgess HJ, Klerman EB, Lewy AJ, Middleton B, Murphy PJ, et al. Measuring melatonin in humans. J Clin Sleep Med 2008 Feb 15;4:66-9.

15. Hutcheon JA, Chiolero A, Hanley JA. Random measurement error and regression dilution bias. BMJ 2010 Jun 26;340: 1402-6.

16. Morgan KE, Cook S, Leon DA, Frost C. Reflection on modern methods: calculating a sample size for a repeatability sub-study to correct for measurement error in a single continuous exposure. Int J Epidemiol 2019 Oct 1;48:1721-6.

17. van den Ouweland JMW, Kema IP. The role of liquid chromatography-tandem mass spectrometry in the clinical laboratory. J Chromatogr B Analyt Technol Biomed Life Sci 2012 Feb 1;883-884:18-32.

18. Taylor AE, Keevil B, Huhtaniemi IT. Mass spectrometry and immunoassay: how to measure steroid hormones today and tomorrow. Eur J Endocrinol 2015 Aug;173:D1-12.

19. Jannetto PJ, Fitzgerald RL. Effective use of mass spectrometry in the clinical laboratory. Clin Chem 2016 Jan;62:92-8.

20. Handelsman DJ. Mass spectrometry, immunoassay and valid steroid measurements in reproductive medicine and science. Hum Reprod 2017 Jun;32:1147-50.

21. de Jong WHA, Graham KS, van der Molen JC, Links TP, Morris MR, Ross HA, et al. Plasma free metanephrine measurement using automated online solid-phase extraction HPLC tandem mass spectrometry. Clin Chem 2007 Sep;53:1684-93.

22. European Medicines Agency. Guideline on bioanalytical method validation [Internet] [cited 2016 Aug 3]. Available from: http:// www.ema.europa.eu/docs/en_GB/document_library/Scientific_ guideline/2011/08/WC500109686.pdf.

23. de Jong WHA, Buitenwerf E, Pranger AT, Riphagen IJ, Wolffenbuttel BHR, Kerstens MN, et al. Determination of reference intervals for urinary steroid profiling using a newly validated GC-MS/MS method. Clin Chem Lab Med 2017 Nov 27;56:103-12.

24. Scholtens S, Smidt N, Swertz MA, Bakker SJ, Dotinga A, Vonk JM, et al. Cohort profile: LifeLines, a three-generation cohort study and biobank. Int J Epidemiol 2015 Aug;44:1172-80.

25. Griefahn B, Remer T, Blaszkewicz M, Bröde P. Long-term stability of 6-hydroxymelatonin sulfate in 24-h urine samples stored at $-20^{\circ} \mathrm{C}$. Endocrine 2001;15:199-202.

26. van Ockenburg SL, Schenk HM, van der Veen A, van Rossum EFC, Kema IP, Rosmalen JGM. The relationship between 63 days of 24-h urinary free cortisol and hair cortisol levels in 10 healthy individuals. Psychoneuroendocrinology 2016 Nov 1;73: 142-7.

27. Fraser GG, Harris EK. Generation and application of data on biological variation in clinical chemistry. Crit Rev Clin Lab Sci 1989 Jan 27;27:409-37.

28. Aarsand AK, Røraas T, Fernandez-Calle P, Ricos C, Díaz-Garzón J, Jonker $\mathrm{N}$, et al. The biological variation data critical appraisal checklist: a standard for evaluating studies on biological variation. Clin Chem 2018 Mar 1;64:501-14.

29. Masri S, Sassone-Corsi P. The emerging link between cancer, metabolism, and circadian rhythms. Nat Med 2018 Dec 6;24: 1795-803.

30. Aldhous ME, Arendt J. Radioimmunoassay for 6-sulphatoxymelatonin in urine using an iodinated tracer. Ann Clin Biochem 1988;25:298-303.

31. Grebe SK, Singh RJ. LC-MS/MS in the clinical laboratory - where to from here? Clin Biochem Rev 2011 Feb;32:5-31.

32. He L, Liu X, Zhang J. Simultaneous quantification of urinary 6-sulfatoxymelatonin and 8-hydroxy-2'-deoxyguanosine using liquid chromatography-tandem mass spectrometry. J Chromatogr B Anal Technol Biomed Life Sci 2018 Sep 15;1095:119-26.

33. Kennaway DJ, Lushington K, Dawson D, Lack L, van den Heuvel C, Rogers N. Urinary 6-sulfatoxymelatonin excretion and aging: new results and a critical review of the literature. J Pineal Res 1999 Nov;27:210-20.

34. Mahlberg R, Tilmann A, Salewski L, Kunz D. Normative data on the daily profile of urinary 6-sulfatoxymelatonin in healthy subjects 
between the ages of 20 and 84 . Psychoneuroendocrinology 2006;31:634-41.

35. Fraser CG. The nature of biological variation. In: Biological variation: from principles to practice. New York: AACC Press; 2001, pp. 1-27.

36. Bojkowski CJ, Arendt J. Annual changes in 6-sulphatoxymelatonin excretion in man. Acta Endocrinol (Copenh) 1988 Apr;117:470-6.

37. Griefahn B, Brode P, Blaszkewicz M, Remer T. Melatonin production during childhood and adolescence: a longitudinal study on the excretion of urinary 6-hydroxymelatonin sulfate. J Pineal Res 2003 Jan;34:26-31.

38. Tetsuo M, Markey SP, Kopin IJ. Measurement of 6-hydroxymelatonin in human urine and its diurnal variations. Life Sci 1980;27:105-9.

39. Fellenberg AJ, Phillipou G, Seamark RF. Specific quantitation of urinary 6-hydroxymelatonin sulphate by gas chromatography mass spectrometry. Biomed Mass Spectrom 1980 Mar;7:84-7.

40. Basler M, Jetter A, Fink D, Seifert B, Kullak-Ublick GA, Trojan A. Urinary excretion of melatonin and association with breast cancer: meta-analysis and review of the literature. Breast Care. S. Karger AG 2014;9:182-7.

41. Xu J, Huang L, Sun GP. Urinary 6-sulfatoxymelatonin level and breast cancer risk: systematic review and meta-analysis. Sci Rep 2017 Dec 1;7:5353.

42. Arendt J. Melatonin: characteristics, concerns, and prospects. J Biol Rhythms 2005 Aug;20:291-303.

Supplementary Material: The online version of this article offers supplementary material (https://doi.org/10.1515/cclm-2020-0455). 